\title{
The Nature of Near Ultraviolet Sources in the GOODS Fields
}

\author{
D. F. de Mello ${ }^{1,2,3}$, T. Dahlen ${ }^{4}$, and Jonathan P. Gardner ${ }^{1}$ \\ ${ }^{1}$ Laboratory for Observational Cosmology, Code 665, Goddard Space Flight Center, Greenbelt, \\ MD 20771. duilia@ipanema.gsfc.nasa.gov \\ ${ }^{2}$ Catholic University of America Washington, DC 20064 \\ ${ }^{3}$ Johns Hopkins University, Baltimore, MD 21218 \\ ${ }^{4}$ Department of Physics, Stockholm University, SE-106 91 Stockholm, Sweden
}

\begin{abstract}
We present the latest results of the survey searching for the nature of UV-selected galaxies in the Great Observatories Origins Deep Survey fields. By searching for UV-bright galaxies at intermediate redshifts $(z \sim 1)$ we aim at understanding the nature of star-forming galaxies and their counterparts at higher-z, the so-called Lyman Break Galaxies.
\end{abstract}

Keywords. galaxies:evolution:formation:starburst

\section{Introduction}

We used the parallel observations taken with HST/WFPC2 in the U-band (F300W) which covered $88 \%$ of the GOODS fields (Giavalisco et al. 2004) to identify sources and selected only objects with GOODS/ACS counterparts. We used the multiwavelength GOODS data to estimate photometric redshifts (Dahlen et al. 2005) and 95 spectroscopic redshifts available through the GOODS collaboration. We used the spectral types obtained by the photometric redshift fitting to identify the starburst galaxies. We have also visually checked all objects and looked for tidal effects and nearby companions.

We present a sample of 268 objects, including 42 stars or AGNs, in the GOODS North and South fields. The analysis of the ACS images (B, V, i, z) of these UV-selected objects reveals that:

(a) Most of the objects are between $0.2<z<0.8$

(b) The majority (45\%) of the galaxies have spectral types of starbursts, however, galaxies of all spectral types are found, including early-types (5\%),

(c) $75 \%$ of the starbursts have tidal tails or show some peculiarity typical of interaction/mergers and $50 \%$ of the starbursts have another galaxy within $5 \times 5$ arcsec;

(d) The bluest galaxies $(U-B<0.2$ and $B-V<0.1)$ are at $1.1<z<1.9$ and have peculiar morphologies that resembles either tadpoles, chains, or double-clump galaxies (see Elmegreen et al. 2005) and half-light radius $1.6 \pm 0.4 \mathrm{kpc}$.

(e) The UV-selected sample has an average rest-frame $\mathrm{M}_{\mathrm{B}}=19.9 \pm 0.1$ which is at least two magnitudes fainter than typical Lyman Break Galaxies.

\section{References}

Dahlen, T., et al. 2005, ApJ, 631, 126

Giavalisco, M., et al., 2004, ApJ, 600, L93.

Elmegreen, D., Elmegreen, B., Rubin, D., \& Schaffer, M., 2005, ApJ, 631, 85 